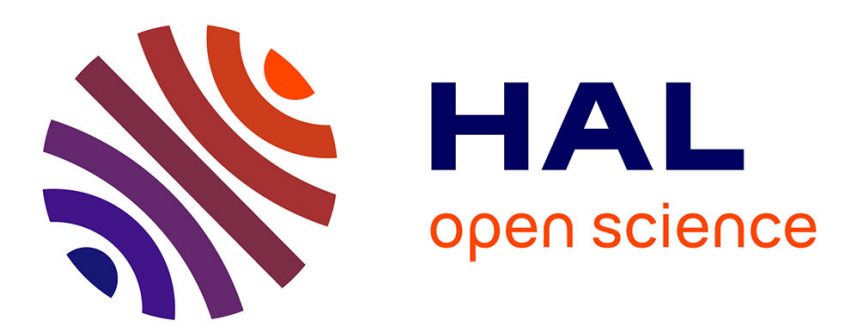

\title{
A Bayesian approach for uncertainty quantification in elliptic Cauchy problem
}

Renaud Ferrier, Mohamed Larbi Kadri, Pierre Gosselet, Hermann G. Matthies

\section{To cite this version:}

Renaud Ferrier, Mohamed Larbi Kadri, Pierre Gosselet, Hermann G. Matthies. A Bayesian approach for uncertainty quantification in elliptic Cauchy problem. Virtual design and validation, Springer, pp.293-308, 2020, 978-3-030-38156-1. 10.1007/978-3-030-38156-1_15 . hal-02344209

\section{HAL Id: hal-02344209 \\ https://hal.science/hal-02344209}

Submitted on 4 Nov 2019

HAL is a multi-disciplinary open access archive for the deposit and dissemination of scientific research documents, whether they are published or not. The documents may come from teaching and research institutions in France or abroad, or from public or private research centers.
L'archive ouverte pluridisciplinaire HAL, est destinée au dépôt et à la diffusion de documents scientifiques de niveau recherche, publiés ou non, émanant des établissements d'enseignement et de recherche français ou étrangers, des laboratoires publics ou privés. 


\title{
A Bayesian approach for uncertainty quantification in elliptic Cauchy problem
}

\author{
Renaud Ferrier and Mohamed Larbi Kadri and Pierre Gosselet and Hermann G. \\ Matthies
}

\begin{abstract}
We study the Cauchy problem in the framework of static linear elasticity and its resolution via the Steklov-Poincaré approach. In the linear Gaussian framework, the straightforward application of Bayes theory leads to formulas allowing to deduce the uncertainty on the identified field from the noise level. We use a truncated Ritz decomposition of the Steklov-Poincaré operator, which reduces the number of degrees of freedom and significantly lowers the computational cost.
\end{abstract}

\section{Introduction}

The boundary data completion problem, or Cauchy problem, that consists, in the framework of static linear elasticity, in computing the unknown loads applied on a boundary of a domain from the knowledge of a measured displacement on another boundary with known prescribed forces, plays an important role in many nondestructive control applications like crack detection [13] or electrocardiography [21].

\footnotetext{
Renaud Ferrier

LMT Cachan, CNRS UMR 8535, ENS Paris-Saclay, 61 Avenue du Président Wilson, 94230 Cachan, France, e-mail: renaud.ferrier@ens-cachan.fr

Mohamed Larbi Kadri

LAMSIN, ENIT, BP 37, 1002, Le Bélvédère, Tunis, Tunisia e-mail: mohamedlarbi.kadri@enit.utm.tn

Pierre Gosselet

LMT Cachan, CNRS UMR 8535, ENS Paris-Saclay, 61 Avenue du Président Wilson, 94230 Cachan, France, e-mail: gosselet@1mt.ens-cachan.fr

Hermann G. Matthies

Institute of Scientific Computing, Technische Universität Braunschweig, Hans-Sommer Str. 65, 38106 Braunschweig, Germany e-mail: wire@tu-bs.de
} 
Under compatibility conditions, the Cauchy problem on elliptic equations has a unique solution. However, it is well-known to be an ill-posed problem in the sense of stability [10]. See the review paper [1] for an optimal logarithmic stability result, and [3] for a spectral analysis of the problem. As a consequence, the solution is very sensitive to the variations in the input. Bayes' theory provides a very suitable framework to quantify uncertainty of the solution.

A large variety of methods have been proposed to deal with this inverse problem. One can cite the optimal control method [17] that consists in minimizing a leastsquare gap, the iterated regularization method [7] introduces an original regularization procedure, the error in constitutive relation method [2] consists in minimizing an energy-like functional, the Dirichlet-to-Neumann method [15] which is a fixed-point method. Some authors proposed to take advantage of the link between harmonic and holomorphic functions [6], others proposed the quasi reversibility method [5], that consists in increasing the order of the PDE. Trefftz methods [16] have also been used to solve the Cauchy problem.

However, as far as the authors know, the literature on the Cauchy problem in stochastic framework is not very widespread. One can note that a Bayesian approach, based on Monte-Carlo sampling has been proposed in [12], and a study with uncertain material parameters has been conducted in [8].

In this work, we use the Steklov-Poincaré method, presented in [4] to solve the Cauchy problem. We propose to invert the linear Steklov-Poincaré system via a Bayesian approach in order to gain access to the uncertainty of the solution. As the problem is linear, in the Gaussian framework one can write formulas that require only to perform one sample for each unknown degree of freedom. However, as we try to identify a field, the number of these degrees of freedom could be, once the problem is discretized, arbitrarily large. As a consequence, the Bayesian inversion may be out of reach. To cure this, we use a truncated Ritz basis of the Steklov-Poincaré operator, as done in [9], in which the problem is diagonal, small and independent to the discretization.

In Section 2, we present the Cauchy problem and the Steklov-Poincare method. Section 3 is dedicated to the presentation of the chosen Bayesian approach. In Section 4, the truncated Ritz decomposition is introduced. In Section 5, a numerical example with a consequent number of degrees of freedom is addressed. The conclusion and perspective of this work are given in Section 6.

\section{The Steklov-Poincaré approach for the Cauchy problem}

\subsection{Forward and Cauchy problems in linear elasticity}

Let $\Omega$ be an open domain of $\mathbb{R}^{d}$ ( $d=2$ or 3 is the dimension of the physical space). $\Gamma_{d}$ (d for Dirichlet) and $\Gamma_{n}$ (n for Neumann) are two disjoint and complementary parts of $\partial \Omega$ (see figure 1a). 


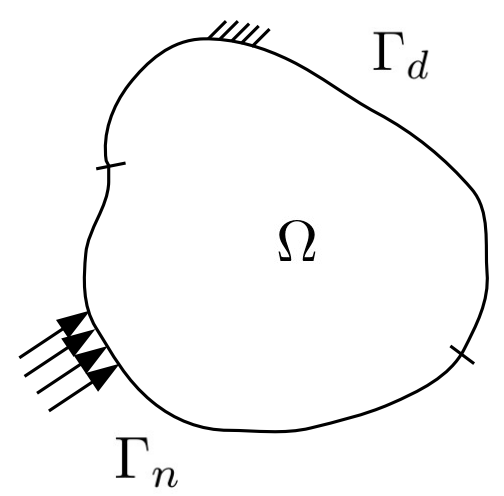

(a) Direct problem

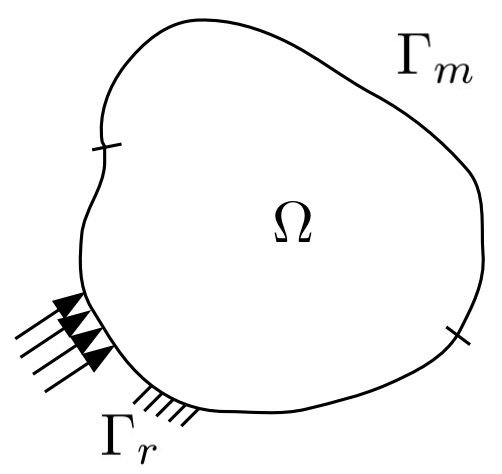

(b) Cauchy problem

Fig. 1: Definition of the domain and its boundaries

We study the equation of static elasticity, on a linear material and under the hypothesis of small perturbations. The main unknown of a direct elasticity problem is the vector field of displacement $\underline{u} \in H^{1}(\Omega)$. In the equations below, $\underline{g}_{0} \in H^{-1}(\Omega)$ is a force density, $\underline{f}_{0} \in H^{-1 / 2}\left(\Gamma_{n}\right)$ is a surface traction and $\underline{u}_{0} \in H^{1 / 2}\left(\Gamma_{d}\right)$ is an imposed displacement. A direct elasticity problem can be written under the following form:

$$
\text { find } \underline{u} \in H^{1}(\Omega) \text { such that: }\left\{\begin{array}{r}
\underline{\operatorname{div}} \underline{\underline{\sigma}}(\underline{u})+\underline{g}_{0}=\underline{0} \text { in } \Omega \\
\underline{u}=\underline{u}_{d} \text { on } \Gamma_{d} \\
\underline{\sigma}(\underline{u}) \cdot \underline{n}=\underline{f}_{n} \text { on } \Gamma_{n}
\end{array}\right.
$$

Where the Cauchy stress tensor $\underline{\underline{\sigma}}$ is related to the linearized strain $\underline{\underline{\varepsilon}}$ by the constitutive equation, characterized by the Hooke tensor $\mathcal{H}$, as follows:

$$
\left\{\begin{array}{l}
\underline{\underline{\sigma}}(\bullet)=\mathcal{H}: \underline{\underline{\varepsilon}}(\bullet) \\
\underline{\underline{\varepsilon}}(\bullet)=\frac{1}{2}\left(\underline{\underline{\operatorname{grad}}}(\bullet)+\underline{\underline{\operatorname{grad}}}(\bullet)^{T}\right)
\end{array}\right.
$$

The Cauchy inverse problem, on the other hand, involves another partitioning of $\partial \Omega$ in two complementary parts $\Gamma_{r}$ ( $\mathrm{r}$ for redundant) and $\Gamma_{m}$ (m for missing, see figure $1 \mathrm{~b})$, it can be formulated as follows:

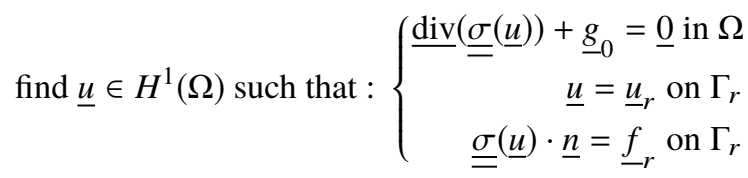


Remark In practice, there can also be some parts of $\partial \Omega$ where simple Dirichlet or Neumann data are given. For simplicity reason, it was chosen, in this article, not to have such boundaries. They would not change the principle of the proposed method.

\subsection{The Steklov-Poincaré method}

Let us define the Steklov operators $\mathcal{S}_{d}$ and $\mathcal{S}_{n}$, and the right-hand sides $\underline{b}_{d}$ and $\underline{b}_{n}$ as follows:

$$
\begin{aligned}
& \mathcal{S}_{d}: \underline{u} \in H^{1 / 2}\left(\Gamma_{m}\right) \\
& \mapsto \mathcal{S}_{d \underline{u}}=\left.\underline{\underline{\sigma}} \underline{\underline{v}} \underline{\underline{v}} \cdot \underline{n}\right|_{\Gamma_{m}} \in H^{-1 / 2}\left(\Gamma_{m}\right),\left\{\begin{aligned}
\underline{\operatorname{div}} \underline{\underline{\sigma}}(\underline{v})) & =\underline{0} \text { in } \Omega \\
\underline{v} & =\underline{0} \text { on } \Gamma_{r} \\
\underline{v} & =\underline{u} \text { on } \Gamma_{m}
\end{aligned}\right. \\
& \mathcal{S}_{n}: \underline{u} \in H^{1 / 2}\left(\Gamma_{m}\right) \\
& \mapsto \mathcal{S}_{n} \underline{u}=\left.\underline{\underline{\sigma}} \underline{\underline{v}} \cdot \underline{n}\right|_{\Gamma_{m}} \in H^{-1 / 2}\left(\Gamma_{m}\right),\left\{\begin{aligned}
\underline{\operatorname{div}}(\underline{\sigma}(\underline{v})) & =\underline{0} \text { in } \Omega \\
\underline{\underline{\sigma}} \underline{\underline{v}}) \cdot \underline{n} & =\underline{0} \text { on } \Gamma_{r} \\
\underline{v} & =\underline{u} \text { on } \Gamma_{m}
\end{aligned}\right. \\
& \underline{b}_{d}=-\left.\underline{\underline{\sigma}}(\underline{v}) \cdot \underline{n}\right|_{\Gamma_{m}} \in H^{-1 / 2}\left(\Gamma_{m}\right),\left\{\begin{aligned}
\underline{\operatorname{div}}(\underline{\sigma}(\underline{v}))+\underline{g}_{0} & =\underline{0} \text { in } \Omega \\
\underline{v} & =\underline{\hat{u}} r \text { on } \Gamma_{r} \\
\underline{v} & =\underline{0} \text { on } \Gamma_{m}
\end{aligned}\right. \\
& \underline{b}_{n}=-\left.\underline{\underline{\sigma}}(\underline{v}) \cdot \underline{n}\right|_{\Gamma_{m}} \in H^{-1 / 2}\left(\Gamma_{m}\right),\left\{\begin{aligned}
\underline{\operatorname{div}}(\underline{\sigma}(\underline{v}))+\underline{g}_{0} & =\underline{0} \text { in } \Omega \\
\underline{\sigma} \underline{\underline{v}}) \cdot \underline{n} & =\underline{\widehat{f}} r \text { on } \Gamma_{r} \\
\underline{v} & =\underline{0} \text { on } \Gamma_{m}
\end{aligned}\right.
\end{aligned}
$$

We can see that $\underline{u}_{m}$ is the trace of the solution of the Cauchy problem (3) on $\Gamma_{m}$ if and only if it respects the following equation:

$$
\left(\mathcal{S}_{d}-\mathcal{S}_{n}\right) \underline{u}_{m}=\underline{b}_{d}-\underline{b}_{n}
$$

This implicit system is then solved with help of a Krylov solver. At each iteration, it is required to apply $\mathcal{S}_{d}$ and $\mathcal{S}_{n}$ which corresponds to solving two direct problems. With this formulation, the ill-posedness of the Cauchy problem is translated in the compactness of the operator $\mathcal{S}_{d}-\mathcal{S}_{n}$ (see [4]).

Once the trace $\underline{u}_{m}$ is found, the resolution of a Dirichlet problem is enough to compute the solution in the entire domain $\Omega$.

Remark This method is named primal Steklov-Poincaré method because the unknown is a displacement field. It is often preconditioned by the operator $\mathcal{S}_{d}^{-1}$ which makes the method an accelerated version of the KMF approach [14]. 
A dual method, that consists in finding the Neumann condition $f_{m}$ that ensures the equality of displacements has been proposed in [13]. Numerical studies, for example in [9], tend to make us think that this dual method is slightly more accurate than the primal one (especially for the determination of the flux on $\Gamma_{m}$ ). Everything that is developed here with the primal method can straightforwardly be transposed to the dual one.

\subsection{Conjugate gradient and Ritz values computation}

Once a discretization method has been chosen, like in our case the finite element method, the system (5) can be written as:

$$
\left(\mathbf{S}_{m, d}-\mathbf{S}_{m, n}\right) \mathbf{u}_{m}=\mathbf{b}_{d}-\mathbf{b}_{n}
$$

$\mathbf{S}_{m, d}$ and $\mathbf{S}_{m, n}$ are Schur complements, the discrete counterparts to Steklov-Poincaré operators; they are Dirichlet-to-Neumann operators on $\Gamma_{m}$ with respectively null displacement or null traction imposed on $\Gamma_{r}$. In our analysis, we will also make use of $\mathbf{S}_{r, d}$ and $\mathbf{S}_{r, n}$, Dirichlet-to-Neumann operators on $\Gamma_{r}$ with respectively null displacement or null traction imposed on $\Gamma_{m}$, and $\mathbf{C}_{r m}$ the rectangular matrix which gives the traction resulting on $\Gamma_{m}$ from an imposed displacement on $\Gamma_{r}$ with null displacement imposed on $\Gamma_{m}$. We have:

$$
\left\{\begin{array}{l}
\mathbf{b}_{d}=-\mathbf{C}_{r m}^{T} \hat{\mathbf{u}}_{r} \\
\mathbf{b}_{n}=-\mathbf{C}_{r m}^{T} \mathbf{S}_{r, d}^{-1} \hat{\mathbf{f}}_{r}
\end{array}\right.
$$

The system (6) was used in [9], where a conjugate gradient algorithm was set up with post-processing of the Ritz values and vectors. The algorithm is reminded in Algorithm 1, note that the formulas for Ritz elements are suitable for multiple right-hand side problems. 


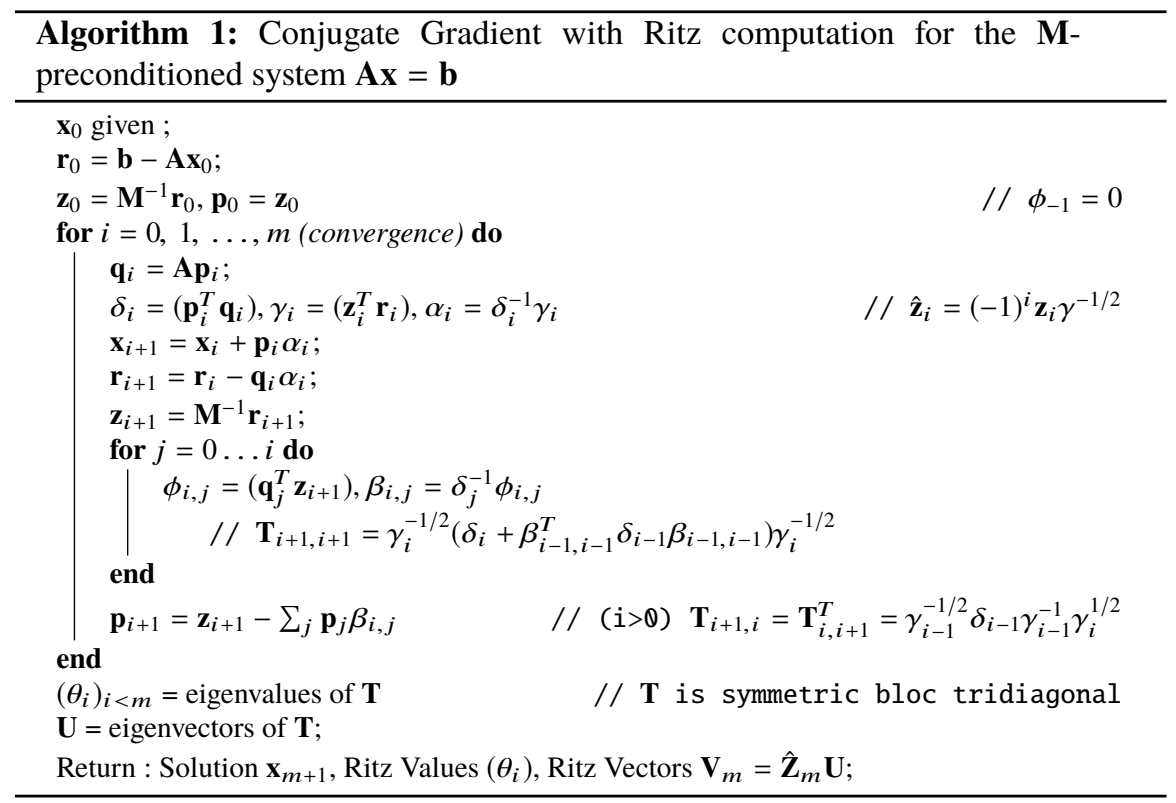

\section{Bayesian inference for the Cauchy problem}

\subsection{Bayes' theory and its application in the linear Gaussian case}

For an extensive review about Bayesian inversion, the reader may refer to [18]. The basic principles are recalled in this section.

Let us suppose that the vectors $\mathbf{x}$ and $\mathbf{b}$ are linked by the linear system $\mathbf{A x}=\mathbf{b}$. Let $\tilde{\mathbf{x}}$ and $\tilde{\mathbf{b}}$ be two real vector-valued random variables. According to Bayes' theorem, it is possible to compute $\mathfrak{p}(\tilde{\mathbf{x}}=\mathbf{x} \mid \tilde{\mathbf{b}}=\mathbf{b})$, the probability that the variable $\tilde{\mathbf{x}}$ have the value $\mathbf{x}$, knowing that $\tilde{\mathbf{b}}$ takes the value $\mathbf{b}$, as follows:

$$
\mathfrak{p}(\tilde{\mathbf{x}}=\mathbf{x} \mid \tilde{\mathbf{b}}=\mathbf{b})=\frac{\mathfrak{p}(\tilde{\mathbf{b}}=\mathbf{b} \mid \tilde{\mathbf{x}}=\mathbf{x}) \mathfrak{p}(\tilde{\mathbf{x}}=\mathbf{x})}{\mathfrak{p}(\tilde{\mathbf{b}}=\mathbf{b})}
$$

In the framework of inverse problems, the probability distribution of $\tilde{x}$ is the unknown and $\mathbf{b}$ is the measurement. We define the following probability functions:

- $\mathfrak{p}(\tilde{\mathbf{x}}=\mathbf{x})$ : the a priori probability, without any information from the measurement, that the variable $\tilde{\mathbf{x}}$ takes the value $\mathbf{x}$. This term ensures the regularizing property of the method;

- $\mathfrak{p}(\tilde{\mathbf{b}}=\mathbf{b})$ : the probability that the variable $\tilde{\mathbf{b}}$ takes the measured value $\mathbf{b}$ (see the remark 3); 
- $\mathfrak{p}(\tilde{\mathbf{b}}=\mathbf{b} \mid \tilde{\mathbf{x}}=\mathbf{x})$ : the probability that the variable $\tilde{\mathbf{b}}$ takes the measured value $\mathbf{b}$, knowing that the variable $\tilde{\mathbf{x}}$ takes the value $\mathbf{x}$. This is function of the accuracy of the measurement;

- $\mathfrak{p}(\tilde{\mathbf{x}}=\mathbf{x} \mid \tilde{\mathbf{b}}=\mathbf{b})$ : the probability that the random variable $\tilde{\mathbf{x}}$ is equal to $\mathbf{x}$, knowing that the measure is $\mathbf{b}$.

Remark In practice, as $\mathfrak{p}(\tilde{\mathbf{b}}=\mathbf{b})$ does not depend on $\mathbf{x}$, it is possible to determine it as the normalization constant ensuring that $\int_{\mathbb{R}^{d}} \mathfrak{p}(\tilde{\mathbf{x}}=\mathbf{x} \mid \tilde{\mathbf{b}}=\mathbf{b}) d \mathbf{x}=1$.

In the case where the problem is linear and where the a priori probabilities are Gaussian, we introduce $C_{\mathbf{b}}$, the covariance matrix of the random variable $\tilde{\mathbf{b}}$ and $C_{\mathbf{x}}^{0}$, the a priori covariance matrix of the random variable $\tilde{\mathbf{x}}$. One has:

$$
\left\{\begin{array}{l}
\mathfrak{p}(\tilde{\mathbf{x}}=\mathbf{x})=\frac{1}{(2 \pi)^{m / 2} \sqrt{\operatorname{det}\left(C_{\mathbf{x}}^{0}\right)}} \exp \left(-\frac{1}{2}\left(\mathbf{x}-\mathbf{x}_{0}\right)^{T}\left[C_{\mathbf{x}}^{0}\right]^{-1}\left(\mathbf{x}-\mathbf{x}_{0}\right)\right) \\
\mathfrak{p}(\tilde{\mathbf{b}}=\mathbf{b} \mid \tilde{\mathbf{x}}=\mathbf{x})=\frac{1}{(2 \pi)^{n / 2} \sqrt{\operatorname{det}\left(C_{\mathbf{b}}\right)}} \exp \left(-\frac{1}{2}(\mathbf{b}-\mathbf{A x})^{T} C_{\mathbf{b}}^{-1}(\mathbf{b}-\mathbf{A x})\right)
\end{array}\right.
$$

It is then possible to analytically determine the a posteriori probability with the following formula (where Cte is a scalar normalization constant):

$$
\begin{aligned}
\mathfrak{p}(\tilde{\mathbf{x}}=\mathbf{x} \mid \tilde{\mathbf{b}}=\mathbf{b})= & C t e \cdot \exp \left(-\frac{1}{2}\left(\mathbf{x}^{T}\left(\mathbf{A}^{T} C_{\mathbf{b}}^{-1} \mathbf{A}+\left[C_{\mathbf{x}}^{0}\right]^{-1}\right) \mathbf{x}\right.\right. \\
& \left.\left.-2\left(\mathbf{x}_{0}^{T}\left[C_{\mathbf{x}}^{0}\right]^{-1}+\mathbf{b}^{T} C_{\mathbf{b}}^{-1} \mathbf{A}\right) \mathbf{x}+\mathbf{x}_{0}^{T}\left[C_{\mathbf{x}}^{0}\right]^{-1} \mathbf{x}_{0}+\mathbf{b}^{T} C_{\mathbf{b}}^{-1} \mathbf{b}\right)\right)
\end{aligned}
$$

It can be remarked that the maximum of likelihood of $\mathbf{x} \mapsto \mathfrak{p}(\tilde{\mathbf{x}}=\mathbf{x} \mid \tilde{\mathbf{b}}=\mathbf{b})$, can be computed as the mean of the a posteriori probability density and satisfies:

$$
\begin{aligned}
\underset{\mathbf{x}}{\arg \max } \mathfrak{p}(\tilde{\mathbf{x}} & =\mathbf{x} \mid \tilde{\mathbf{b}}=\mathbf{b})=\underset{\mathbf{b}}{\mathbf{b}}(\tilde{\mathbf{x}})=\underset{\mathbf{x}}{\arg \min }\left(\mathbf{x}^{T}\left(\mathbf{A}^{T} C_{\mathbf{b}}^{-1} \mathbf{A}+\left[C_{\mathbf{x}}^{0}\right]^{-1}\right) \mathbf{x}\right. \\
& \left.-2\left(\mathbf{x}_{0}^{T}\left[C_{\mathbf{x}}^{0}\right]^{-1}+\mathbf{b}^{T} C_{\mathbf{b}}^{-1} \mathbf{A}\right) \mathbf{x}+\mathbf{x}_{0}^{T}\left[C_{\mathbf{x}}^{0}\right]^{-1} \mathbf{x}_{0}+\mathbf{b}^{T} C_{b}^{-1} \mathbf{b}\right)
\end{aligned}
$$

This value, that will be noted $\mathbf{x}_{\text {post }}$ is thus the solution to the following linear problem:

$$
\left(\mathbf{A}^{T} C_{\mathbf{b}}^{-1} \mathbf{A}+\left[C_{\mathbf{x}}^{0}\right]^{-1}\right) \mathbf{x}_{\text {post }}=\left[C_{\mathbf{x}}^{0}\right]^{-1} \mathbf{x}_{0}+\mathbf{A}^{T} C_{\mathbf{b}}^{-1} \mathbf{b}
$$

In this case, the mean of the a posteriori probability density is the solution of a preconditioned and regularized mean-square system. The particularity of the Bayesian approach is that we also have access to the covariance matrix, denoted $C_{\mathbf{x}, \text { post }}$, that contains, in the Gaussian case, all the information about the probability density function :

$$
C_{\mathbf{x}, \text { post }}^{-1}=\mathbf{A}^{T} C_{\mathbf{b}}^{-1} \mathbf{A}+\left[C_{\mathbf{x}}^{0}\right]^{-1}
$$

It can be remarked that this resolution is equivalent to the Kalman filtering in the linear Gaussian case, and with only one observation. Kalman filtering consists in defining a gain $\mathbf{K}$ and computing the mean and the a posteriori correlation matrix 
as follows:

$$
\left\{\begin{array}{l}
\mathbf{K}=C_{\mathbf{x}}^{0} \mathbf{A}^{T}\left(\mathbf{A} C_{\mathbf{x}}^{0} \mathbf{A}^{T}+C_{\mathbf{b}}\right)^{-1} \\
\mathbf{x}_{\text {post }}=\mathbf{x}_{0}+\mathbf{K}\left(\mathbf{b}-\mathbf{A} \mathbf{x}_{0}\right) \\
C_{\mathbf{x}, \text { post }}=(\mathbf{I}-\mathbf{K A}) C_{\mathbf{x}}^{0}
\end{array}\right.
$$

one can show that $\mathbf{x}_{\text {post }}$ and $C_{\text {post }}$ satisfy the relations (12) and (13). This way to compute the correlation is more reliable than the previous one because it does not request to perform inversions of correlation matrices, that are possibly ill-conditionned.

\subsection{Application to the Cauchy problem}

In the case of the Cauchy problem, we focus on the Steklov-Poincaré system (6). The first step consists in propagating the uncertainties from $\hat{\mathbf{f}}_{r}$ and $\hat{\mathbf{u}}_{r}$ to $\mathbf{b}_{d}$ and $\mathbf{b}_{n}$. We define the correlation matrices associated to these quantities $C_{\hat{\mathbf{f}}_{r}}, C_{\hat{\mathbf{u}}_{r}}, C_{\mathbf{b}_{d}}$ and $C_{\mathbf{b}_{n}}$. By direct propagation of the uncertainties, one gets:

$$
\left\{\begin{array}{l}
C_{\mathbf{b}_{d}}=\mathbf{C}_{r m}^{T} C_{\hat{\mathbf{u}}_{r}} \mathbf{C}_{r m} \\
C_{\mathbf{b}_{n}}=\mathbf{C}_{r m}^{T} \mathbf{S}_{r, n}^{-1} C_{\hat{\mathbf{f}}_{r}} \mathbf{S}_{r, n}^{-1} \mathbf{C}_{r m}
\end{array}\right.
$$

One can remark that the computation of $C_{\mathbf{b}_{d}}$ and $C_{\mathbf{b}_{n}}$ necessitates to assemble $\mathbf{C}_{r m}$ and $\mathbf{C}_{r m}^{T} \mathbf{S}_{r, n}^{-1}$, which is equivalent to solving one direct problem for each degree of freedom on $\Gamma_{m}$. This operation is extremely costly in term of CPU time.

The covariance matrix of the right-hand side $\mathbf{b}_{d}-\mathbf{b}_{n}$ is the sum of both contributions : $C_{\mathbf{b}_{d}-\mathbf{b}_{n}}=C_{\mathbf{b}_{d}}+C_{\mathbf{b}_{n}}$. The a priori mean of the unknown, $\mathbf{u}_{m}^{0}$ and its covariance matrix, $C_{\mathbf{u}_{m}}^{0}$ are then introduced. From all these pieces of information, we seek to determine the a posteriori mean, denoted by $\mathbf{u}_{m}$ and its covariance matrix, $C_{\mathbf{u}_{m}}$.

The Kalman formula (14) gives:

$$
\left\{\begin{aligned}
\mathbf{K}= & C_{\mathbf{u}_{m}}^{0}\left(\mathbf{S}_{m, d}-\mathbf{S}_{m, n}\right) \\
& \left(\left(\mathbf{S}_{m, d}-\mathbf{S}_{m, n}\right) C_{\mathbf{u}_{m}}^{0}\left(\mathbf{S}_{m, d}-\mathbf{S}_{m, n}\right)+C_{\mathbf{b}_{d}-\mathbf{b}_{n}}\right)^{-1} \\
\mathbf{u}_{m}= & \mathbf{u}_{m}^{0}+\mathbf{K}\left(\mathbf{b}_{d}-\mathbf{b}_{n}-\left(\mathbf{S}_{m, d}-\mathbf{S}_{m, n}\right) \mathbf{u}_{m}^{0}\right) \\
C_{\mathbf{u}_{m}}= & C_{\mathbf{u}_{m}}^{0}-\mathbf{K}\left(\mathbf{S}_{m, d}-\mathbf{S}_{m, n}\right) C_{\mathbf{u}_{m}}^{0}
\end{aligned}\right.
$$

The characterization of the a priori uncertainty on $\mathbf{u}_{m}^{0}$ and on the data $\hat{\mathbf{u}}_{r}$ and $\hat{\mathbf{f}}_{r}$ is a crucial point. The uncertainty on $\mathbf{u}_{m}^{0}$ gives the interval in which the solution will be searched for; as a consequence, one can propose to determine it from the magnitude of a rough solution of the problem. In many applications, $\hat{\mathbf{f}}_{r}$ is in fact a null force on a free boundary, and as a consequence, the associated uncertainty can be taken as zero. As for $\hat{\mathbf{u}}_{r}$, its uncertainty can sometimes be estimated from the knowledge of the precision of the measurement mean. 
The application of the above formulas to determine the probability density function of the solution necessitates to explicitly know the operator $\mathbf{S}_{m, d}-\mathbf{S}_{m, n}$. Such an approach is possible in a case where the number of degrees of freedom is reasonably small, but it is unusable in the case of a full field identification. Next section shows how Ritz analysis makes it possible to apply these formulas.

\section{Reduction by Ritz modes}

Let us suppose that a Ritz analysis has been done on the system, leading to the basis of Ritz vectors $\mathbf{V}$, as explained in Part 4. Let $\mathbf{u}_{m}=\mathbf{V} \tilde{\mathbf{x}}$ and $\tilde{\mathbf{y}}=\mathbf{V}^{T}\left(\mathbf{b}_{d}-\mathbf{b}_{n}\right)$. We have:

$$
\mathbf{V}^{T}\left(\mathbf{S}_{m, d}-\mathbf{S}_{m, n}\right) \mathbf{V}=\Theta \text { diagonal, } \quad \mathbf{V}^{T} \mathbf{M V}=\mathbf{I}, \quad \Theta \tilde{\mathbf{x}}=\tilde{\mathbf{y}}
$$

The exact same analysis as in Section 3 is made on this reduced system. One has first to propagate the uncertainties on $\hat{\mathbf{f}}_{r}$ and $\hat{\mathbf{u}}_{r}$ to $\tilde{\mathbf{y}}$ and the uncertainties on $\mathbf{u}_{m}^{0}$ to $\tilde{\mathbf{x}}$. The corresponding covariance matrices are denoted by $C_{\tilde{\mathbf{y}}}$ and $C_{\tilde{\mathbf{x}}}^{0}$.

$$
\left\{\begin{aligned}
C_{\mathbf{y}_{d}} & =\mathbf{V}^{T} \mathbf{C}_{r m}^{T} C_{\hat{\mathbf{u}}_{r}} \mathbf{C}_{r m} \mathbf{V} \\
C_{\mathbf{y}_{n}} & =\mathbf{V}^{T} \mathbf{C}_{r m}^{T} \mathbf{S}_{r, n}^{-1} C_{\hat{\mathbf{f}}_{r}} \mathbf{S}_{r, n}^{-1} \mathbf{C}_{r m} \mathbf{V} \\
C_{\tilde{\mathbf{y}}} & =C_{\mathbf{y}_{d}}+C_{\mathbf{y}_{n}} \\
C_{\tilde{\mathbf{x}}}^{0} & =\mathbf{V}^{T} \mathbf{M} C_{\mathbf{u}_{m}}^{0} \mathbf{M V}
\end{aligned}\right.
$$

The computation of $\mathbf{C}_{r m} \mathbf{V}$ and $\mathbf{S}_{r, n}^{-1} \mathbf{C}_{r m} \mathbf{V}$ is much less complex than previously. Assembling $\mathbf{C}_{r m} \mathbf{V}$ requires solving problems on which Dirichlet conditions of value $\mathbf{V}_{i}$ are applied on $\Gamma_{m}$ and a zero Dirichlet condition is imposed on $\Gamma_{r}$. The assembly of $\mathbf{S}_{r, n}^{-1} \mathbf{C}_{r m} \mathbf{V}$ requires solving problems on which Dirichlet conditions of value $\mathbf{V}_{i}$ are imposed on $\Gamma_{m}$ and a zero Neumann condition is imposed on $\Gamma_{r}$. Each computation requires solving as many direct problems as the number of Ritz modes in the chosen basis.

What is more, one can remark that these direct problems are the same as those that permit to evaluate $\mathbf{S}_{m, d}$ and $\mathbf{S}_{m, n}$ (except that the field is recovered on the boundary $\Gamma_{r}$ ). As a consequence, it is possible to compute $\mathbf{C}_{r m} \mathbf{V}$ and $\mathbf{S}_{r, n}^{-1} \mathbf{C}_{r m} \mathbf{V}$ at very small cost during the iterations of the conjugate gradient (see Algorithm 1). One needs to store the corresponding vectors after each evaluation of $\mathbf{S}_{m, d} \mathbf{p}_{i}$ and $\mathbf{S}_{m, n} \mathbf{p}_{i}$. One uses the fact that for $i>0, \mathbf{C}_{r m} \mathbf{z}_{i}=\mathbf{C}_{r m} \mathbf{p}_{i}+\sum_{j} \mathbf{C}_{r m} \mathbf{p}_{j} \beta_{i-1, j}$ (same thing for $\mathbf{S}_{r, n}^{-1} \mathbf{C}_{r m} \mathbf{Z}_{i}$ ). $\mathbf{C}_{r m} \mathbf{Z}$ and $\mathbf{S}_{r, n}^{-1} \mathbf{C}_{r m} \mathbf{Z}$ are then transformed the same way as $\mathbf{Z}$ in order to build the required operators. The vectors that compose $\mathbf{M V}$ can also be built during the iterations of the conjugate gradient on the same model as $\mathbf{V}$ by writing $\hat{\mathbf{r}}_{i}=(-1)^{i} \mathbf{r}_{i} \gamma^{-1 / 2}$ and $\mathbf{M V}=\hat{\mathbf{R}} \mathbf{U}$ in Algorithm 1 .

This leads to: 


$$
\left\{\begin{aligned}
\mathbf{K} & =C_{\tilde{\mathbf{x}}}^{0} \Theta\left(\Theta C_{\tilde{\mathbf{x}}}^{0} \Theta+C_{\tilde{\mathbf{y}}}\right)^{-1} \\
\tilde{\mathbf{x}} & =\tilde{\mathbf{x}}^{0}+\mathbf{K}\left(\tilde{\mathbf{y}}-\Theta \tilde{\mathbf{x}}^{0}\right) \\
C_{\tilde{\mathbf{x}}} & =C_{\tilde{\mathbf{x}}}^{0}-\mathbf{K} \Theta C_{\tilde{\mathbf{x}}}^{0}
\end{aligned}\right.
$$

All the operators implied in these relations have the same size as the truncated Ritz basis. Consequently, all the operations have a very small cost.

Finally, one propagates the uncertainties on $\tilde{\mathbf{x}}$ to $\mathbf{u}_{m}$ thanks to the approximation $C_{\mathbf{u}_{m}} \simeq \mathbf{V} C_{\tilde{\mathbf{x}}} \mathbf{V}^{T}$ (at this stage, a part of the uncertainty is neglected because of the truncation of the Ritz basis) and $\mathbf{u}_{m}=\mathbf{V} \tilde{\mathbf{x}}$. For the determination of the force $\mathbf{f}_{m}$, we can define it as $\mathbf{f}_{m}^{d}=\mathbf{S}_{m, d} \mathbf{u}_{m}-\mathbf{b}_{d}$ or $\mathbf{f}_{m}^{n}=\mathbf{S}_{m, n} \mathbf{u}_{m}-\mathbf{b}_{n}$ (they are supposed to be equal if $\mathbf{u}_{m}$ is equal to the reference solution). If $\mathbf{b}_{n}$ is supposed to be noiseless, the second expression is probably better, and thus $C_{\mathbf{f}_{m}}=\mathbf{S}_{m, n} \mathbf{V} C_{\tilde{\mathbf{x}}} \mathbf{V}^{T} \mathbf{S}_{m, n}$. As previously, the determination of $\mathbf{S}_{m, n} \mathbf{V}$ requires solving as many direct problems as the number of vectors in the truncated Ritz basis, and as previously, this matrix can be assembled during the conjugate gradient iterations by storing the vector $\mathbf{S}_{m, n} \mathbf{p}_{i}$ and doing a few operations on it. However, the application of $\mathbf{S}_{m, n}$ can be interpreted in this context as a derivation operation, that is well-known for being ill-conditioned. If one considers that the solution $\mathbf{u}_{m}$ is noisy, the flux $\mathbf{f}_{m}$ is then likely to be even noisier. For that reason, its determination should preferably be done with another regularization procedure, which gets out of the scope of this article. For that reason, in the numerical part, only the determination of $\mathbf{u}_{m}$ is studied.

The procedure is summed up in Algorithm 2.

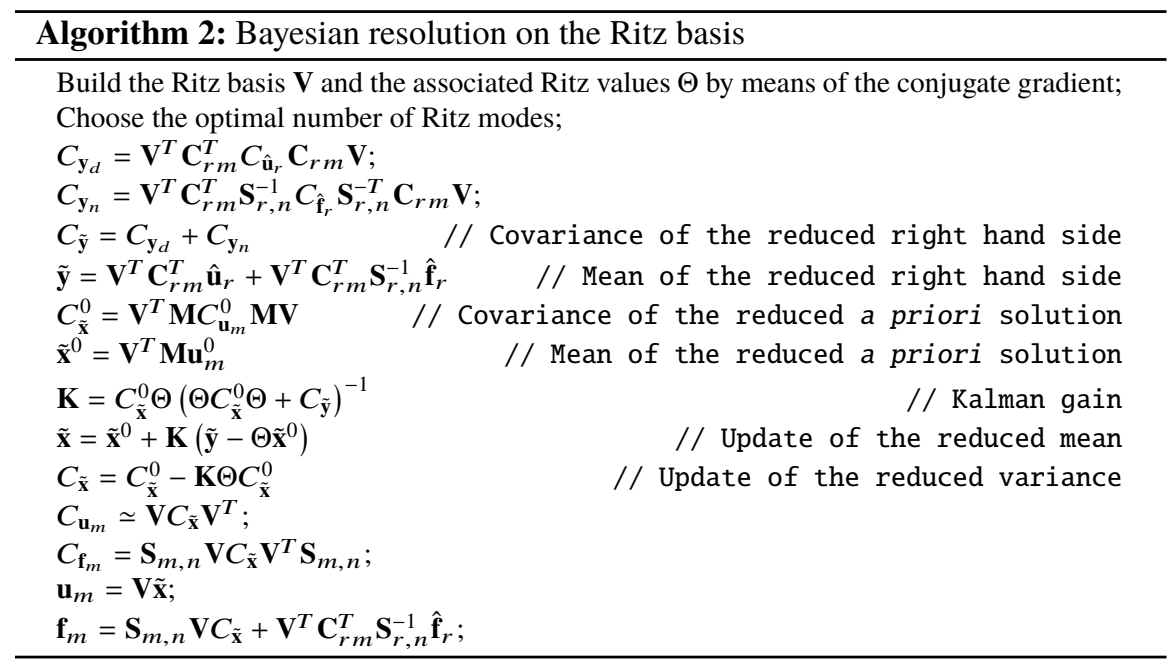

The only computationally expensive step is the computation of the Ritz values and vectors, that is equivalent to the cost of the deterministic resolution by the Steklov-Poincaré method. All the other operations are either done on small operators, 
or doable at very small extra cost during the iterations of the conjugate gradient algorithm.

Remark As for all Bayesian methods, the results obtained are very dependent of the choice of the a priori probability density function. In the case when this function is not available, one can imagine a variant of the method where $\mathbf{u}_{m}$ is determined by the Steklov-Poincare method and the covariance is obtained with a propagation of the uncertainties $C_{\tilde{\mathbf{x}}}=\Theta^{-1} C_{\tilde{\mathbf{y}}} \Theta^{-1}$, that corresponds to the formula (19) in the case of equiprobable a priori information on $\mathbb{R}^{n}$ (with $n$ the dimension of $\tilde{\mathbf{x}}$ ).

Remark The truncation of the Ritz basis is a sine qua non condition to make the computation of the uncertainty on the solution affordable in the cases with a high number of unknowns. However, this is done at the cost of a simplification of the problem, and thus a loss of accuracy. Numerically, it was observed that the uncertainty increases with the number of Ritz modes, but tends to stagnate because the contribution of the highest order Ritz modes is erased by the regularizing effect of the Bayesian approach. The choice of the relevant number of modes (which does not match the one given by the discrete Picard condition [11,9]), and the evaluation of the resulting imprecision are important questions that are not investigated in this study.

\section{Numerical example}

We study a hollow sphere with an internal inhomogeneous pressure $p=a x^{2}+b$ with $a=1 \mathrm{~N} \cdot \mathrm{mm}^{-4}$ and $b=10 \mathrm{~N} \cdot \mathrm{mm}^{-2}$. The Cauchy problem consists in determining the displacement and pressure on the internal boundary from the observation of the displacement on the outer Neumann-free boundary.

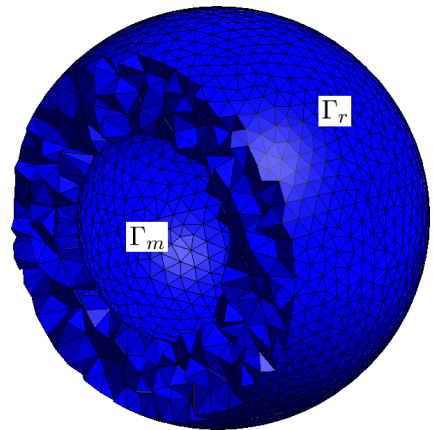

(a) Domain (clipped) and its mesh

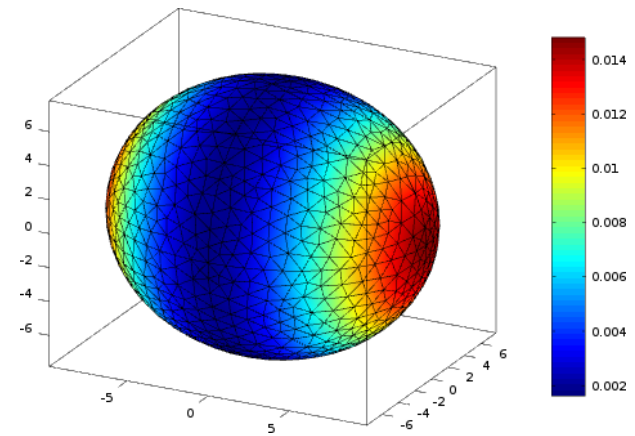

(b) Amplitude of displacement and deformed mesh of $\Gamma_{m}$

Fig. 2: Domain and its mesh 
The interior radius is $R_{1}=8 \mathrm{~mm}$ and the exterior radius is $R_{2}=12 \mathrm{~mm}$. We consider a uncorrelated Gaussian noise of amplitude $20 \%$ on the data $\hat{\mathbf{u}}_{r}$. The isotropic linear elasticity coefficients are Young modulus $E=210000 \mathrm{MPa}$ and Poisson ratio $v=0.3$. We use a tetrahedral mesh of which edges length is approximately $l=1$ $\mathrm{mm}$. The total number of degrees of freedom is 15156.6357 of them are on $\Gamma_{r}$ and 3042 on $\Gamma_{m}$, which corresponds to the number of unknowns of the inverse problem.

We illustrate on Figure 3 the fact that the truncated Ritz decomposition is relatively independent of the mesh size. Three meshes are studied: the first one has a size $l=1.5$ $\mathrm{mm}$, which results in a total number of degrees of freedom of 5814, 3033 of which are on $\Gamma_{r}$ and 1503 on $\Gamma_{m}$. The second discretization is the one that will be used for the uncertainty quantification. The third mesh has a size $l=0.75 \mathrm{~mm}$, which results in a total number of degrees of freedom of 33612, 12585 of which are on $\Gamma_{r}$ and 5088 on $\Gamma_{m}$. One can observe that the first Ritz values $\Theta$, the terms of the projection of the right hand side $\mathbf{V}^{T}\left(\mathbf{b}_{d}-\mathbf{b}_{n}\right)$ and the terms of the projection of the solution $\tilde{\mathbf{x}}$ are quite similar for the different mesh sizes.

The next step consists in choosing the number of Ritz modes used for the resolution. In [9], a criterion similar to the discrete Picard condition [11] was proposed. This criterion leads to use about 50 modes. However, it was numerically observed that this number of modes leads to errors on the solution that are much higher than the uncertainty estimation.

Indeed, it was observed that around the optimal value of 50 modes, the error has a quite uniform amplitude, but the more modes there are, the lower the error due to neglected modes is and the higher the error due to noise on the data is. Consequently, in order to quantify the error thanks to a reduced model, it is better that the error due to the neglected modes is small with respect to the error due to the noise. For that reason, the chosen number of modes needs to be as large as possible. It was chosen to use 77 modes as, at this point, the increase of the components of $\tilde{\mathbf{x}}$ seems to be more regular, which let us think that it is governed by the white noise (see the vertical bar on Figure 3b).

The chosen a priori probability has a null mean value and all the degrees of freedom are uncorrelated. The variance is determined by the amplitude of the solution of the problem by the deterministic Steklov-Poincare method projected on the 50 first Ritz modes (in conformity with the discrete Picard condition). One uses Algorithm 2 to compute the mean $\mathbf{u}_{m}$ and its variance matrix $C_{\mathbf{u}_{m}}$. From this variance matrix, one extracts the diagonal terms, that correspond to the uncertainty on $\mathbf{u}_{m}$. On Figure 4, the variance on the component $u_{z}$ is compared to the error on this component. It can be noticed that the variance gives indeed a relevant idea of the uncertainty level. In order to achieve a more quantitative comparison, both are plotted on the meridian of the sphere of equation $x=0$ on Figure 5 .

The same procedure has been applied on a thicker domain with $R_{1}=5 \mathrm{~mm}$, $R_{2}=15 \mathrm{~mm}$ and $l=2 \mathrm{~mm}$. This results in much fewer degrees of freedom on $\Gamma_{m}$ (348), without significantly modifying the Ritz spectrum. The number of modes used for the Bayesian inversion remains the same (77), and the noise is decreased to $5 \%$. The maps of the error and estimated standard deviation are presented on Figure 6 , and their comparison on the meridian is displayed on Figure 7. On this last figure, 


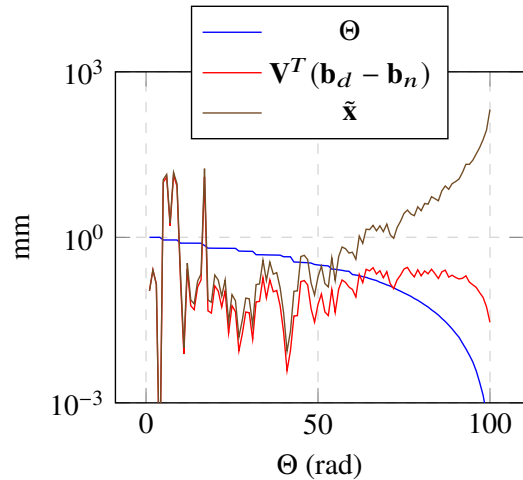

(a) $l=1.5 \mathrm{~mm}$

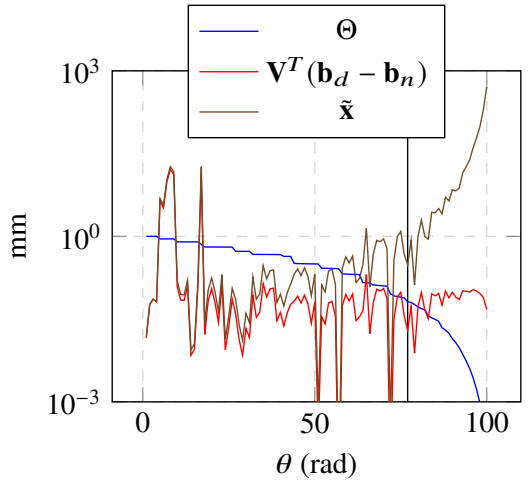

(b) $l=1 \mathrm{~mm}$ (default). The vertical bar marks the chosen number of modes for the Bayesian resolution

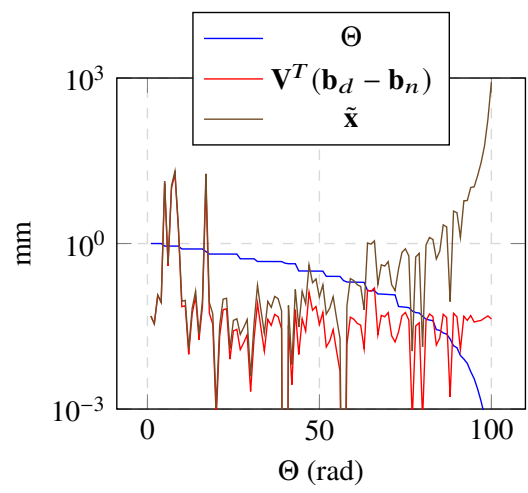

(c) $l=.75 \mathrm{~mm}$

Fig. 3: Ritz values, projection of the right-hand side and of the solution on the Ritz basis for different mesh size $l$

the errors due to two realizations of the random noise are also plotted. Note that the computed standard deviation is not strictly the same for those two realization of the random noise (although being very close) because this field is computed via the Ritz modes, that slightly depend on the right hand side, that is impacted by the noise.

\section{Conclusion and perspectives}

In this article, a simple Bayesian approach has been applied to solve the Cauchy problem on an elliptic PDE. As we seek to identify a continuous field, which means 


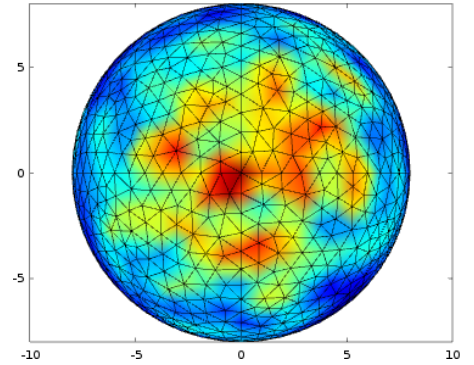

(a) Computed standard deviation

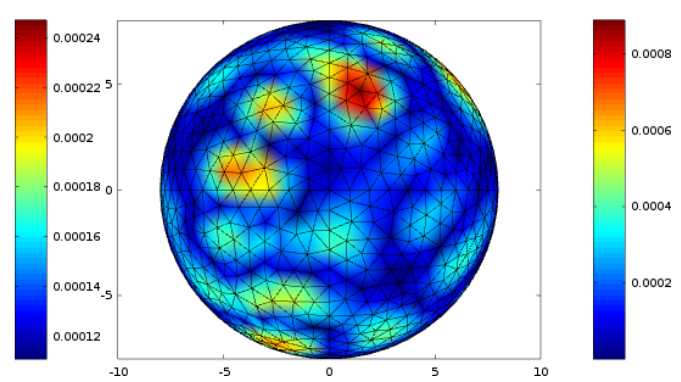

(b) Error on the Bayesian identification

Fig. 4: Error and standard deviation on $u_{z}($ noise $=20 \%)$

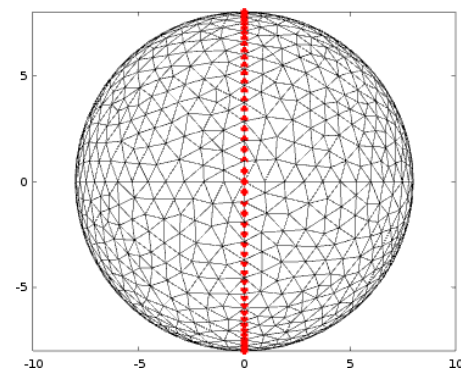

(a) Localization of the meridian

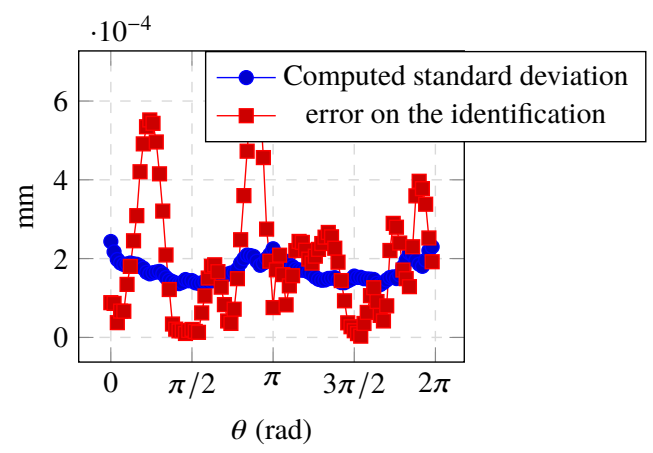

(b) Error and standard deviation on the meridian

Fig. 5: Error and standard deviation on $u_{z}$ (noise $=20 \%$ ) on the meridian

that the number of degrees of freedom can be arbitrarily large, a Ritz decomposition of the involved operator has been used to work in a space that is independent of the discretization.

The chosen approach has shown good results concerning the estimation of the uncertainties on the solution provided two requirements are satisfied. First, the noise level should be sufficiently high in order to make it possible to consider it to be the dominant source of error in the resolution of the discretized system. On some geometries, the Cauchy problem is indeed so ill-posed that the numerical truncation noise (whose impact is much harder to quantify) is the main source of error on the solution. The second requirement is that the number of Ritz modes should be sufficient to ensure that the contribution of the neglected part of the spectrum to the error on the solution is small. The determination of the minimal number of modes to meet this requirement is still an open question.

What is more, one can remark that nothing prevents to apply the proposed procedure on other inverse problems solved with a Conjugate Gradient algorithm. How- 


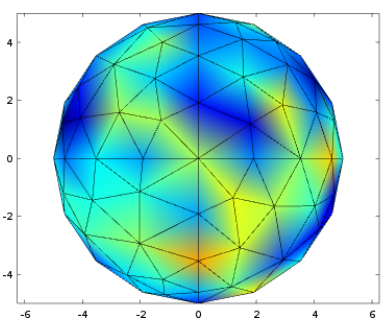

(a) Computed standard deviation

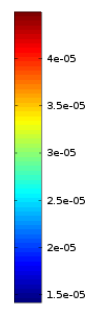

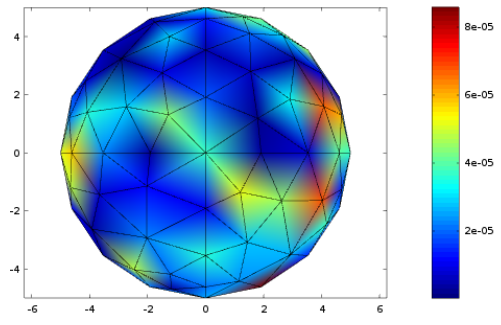

(b) Error on the Bayesian identification

Fig. 6: Error and standard deviation on $u_{z}$ (thicker sphere, noise $=5 \%$ )

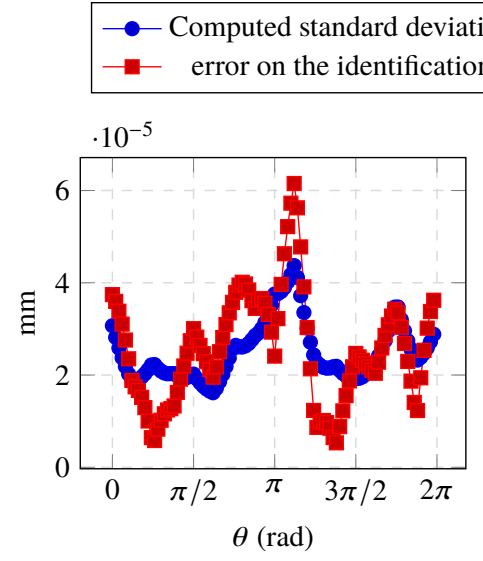

(a) First realization
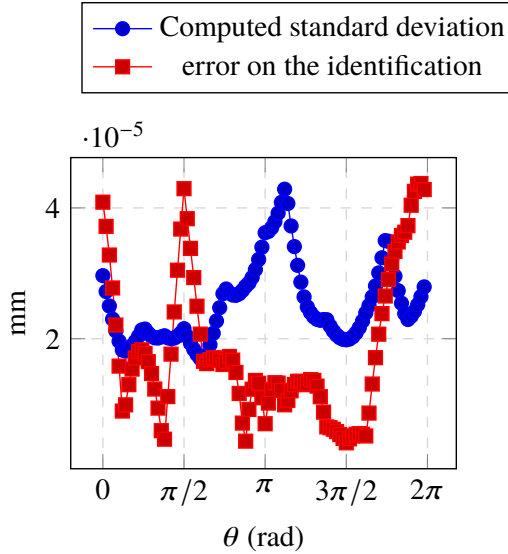

(b) Second realization

Fig. 7: Error and standard deviation on $u_{z}$ (thicker sphere, noise $=5 \%$ ) on the meridian

ever, this study has been conducted in the linear Gaussian framework, which tends to facilitate a lot the development of CPU efficient Bayesian methods. However, in nonlinear or non-Gaussian cases, this work could be extended thanks to the sampling-free Bayesian method presented in [20] and [19], that uses a polynomial chaos expansion to represent non-Gaussian probabilities.

Acknowledgements This work has been accomplished thanks to the ViVaCE - IRTG 1627 program. 


\section{References}

1. Alessandrini, G., Rondi, L., Rosset, E., and Vessella, S. The stability for the Cauchy problem for elliptic equations. Inverse problems 25, 12 (2009), 123004.

2. Andrieux, S., Baranger, T. N., And Ben Abda, A. Solving Cauchy problems by minimizing an energy-like functional. Inverse problems 22, 1 (2006), 115.

3. Ben Belgacem, F. Why is the Cauchy problem severely ill-posed? Inverse Problems 23, 2 (2007), 823-836.

4. Ben Belgacem, F., and El Fekin, H. On Cauchy's problem: I. A variational Steklov-Poincaré theory. Inverse Problems 21, 6 (2005), 1915.

5. Bourgeors, L. A mixed formulation of quasi-reversibility to solve the Cauchy problem for Laplace's equation. Inverse problems 21, 3 (2005), 1087.

6. Chatbane, S., Jaoua, M., And Leblond, J. Parameter identification for Laplace equation and approximation in Hardy classes. Journal of Inverse and Ill-posed Problems jiip 11, 1 (2003), $33-57$.

7. Cimetiere, A., Delvare, F., Jaoua, M., and Pons, F. Solution of the Cauchy problem using iterated Tikhonov regularization. Inverse Problems 17, 3 (2001), 553-570.

8. Faverjon, B., Puig, B., ANd Baranger, T. Identification of boundary conditions by solving Cauchy problem in linear elasticity with material uncertainties. Computers \& Mathematics with Applications 73, 3 (2017), 494-504.

9. Ferrier, R., Kadri, M. L., and Gosselet, P. The Steklov-Poincaré technique for data completion: Preconditioning and filtering. International Journal for Numerical Methods in Engineering 116, 4, 270-286.

10. Hadamard, J. Lectures on Cauchy's problem in linear partial differential equations, vol. 37. Yale University Press, 1923.

11. Hansen, P. C. The discrete Picard condition for discrete ill-posed problems. BIT Numerical Mathematics 30, 4 (1990), 658-672.

12. JiN, B., AND Zou, J. A Bayesian inference approach to the ill-posed Cauchy problem of steady-state heat conduction. International Journal for Numerical Methods in Engineering 76, 4 (2008), 521-544.

13. Kadri, M. L., Ben Abdallah, J., And Baranger, T. N. Identification of internal cracks in a three-dimensional solid body via Steklov-Poincaré approaches. Comptes Rendus Mécanique 339, 10 (2011), 674-681.

14. Kozlov, V. A., AND MAZ'ya, V. G. Iterative procedures for solving ill-posed boundary value problems that preserve the differential equations. Algebra $i$ Analiz 1, 5 (1989), 144-170.

15. Kozlov, V. A., Maz'ya, V. G., And Fomin, A. An iterative method for solving the Cauchy problem for elliptic equations. Zhurnal Vychislitel'noi Matematiki i Matematicheskoi Fiziki 31, 1 (1991), 64-74.

16. LiU, C.-S., ET AL. A highly accurate MCTM for inverse Cauchy problems of Laplace equation in arbitrary plane domains. CMES: Computer Modeling in Engineering \& Sciences 35, 2 (2008), 91-111.

17. Marin, L., Hìo, D. N., And Lesnic, D. Conjugate gradient-boundary element method for the Cauchy problem in elasticity. Quarterly Journal of Mechanics and Applied Mathematics 55, 2 (2002), 227-247.

18. Matthies, H. G. Uncertainty quantification and b ayesian inversion. Encyclopedia of Computational Mechanics Second Edition (2017), 1-51.

19. Matthies, H. G., Zander, E., Rosić, B. V., Litvinenko, A., and Pajonk, O. Inverse problems in a Bayesian setting. In Computational Methods for Solids and Fluids. Springer, 2016, pp. 245 286.

20. Rosic, B. V., Litvinenko, A., Pajonk, O., and Matthies, H. G. Direct Bayesian update of polynomial chaos representations. Journal of Computational Physics (2011).

21. Zemzemi, N. A domain decomposition approach in the electrocardiography inverse problem. In Domain Decomposition Methods in Science and Engineering XXII. Springer, 2016, pp. 641647. 